\title{
¿Qué ha cambiado con esta crisis? El mainstreaming de género en la encrucijada
}

\author{
Eva ALFAMA \\ Universitat Autònoma de Barcelona \\ eva alfama@yahoo.com \\ Marta CRUELLS \\ Universitat Autònoma de Barcelona. \\ martama6@yahoo.es \\ María DE LA FUENTE \\ Observatorio IQ
}

Recibido: 25.08.2014

Aceptado: 20.01.2015

\section{RESUMEN:}

Este artículo se centra en reflexionar acerca de los retos a los que se enfrenta la estrategia del gender mainstreaming o transversalidad de género en el contexto económico y político actual, recurriendo a la literatura feminista sobre las políticas de igualdad y sobre la crisis económica. Para ello, se revisan brevemente los logros conseguidos y los límites encontrados en el desarrollo de las políticas de igualdad de género hasta el momento actual -a nivel europeo y estatal-, y se explora la dimensión de género tanto de la crisis económica como de las políticas implementadas para dar respuesta a la misma. De este modo se identifican los retos y obstáculos que la gobernanza económica y política actual plantea a la hora de implantar la transversalidad de género en el Estado español, y se discute sobre las posibilidades de actuación existentes, planteando algunas preguntas acerca de la necesidad de replantear las estrategias e instrumentos de las políticas de igualdad en este nuevo escenario.

Palabras clave: Crisis económica; Políticas de igualdad; Igualdad de género; Transversalidad de género; Democratización.

\section{Rethinking Gender Mainstreaming in the era of austerity}

\begin{abstract}
:
This paper address the implications for the Gender Mainstreaming strategy of the present economic and political crisis, at both EU and Spanish level. In the first place the main achievements and shortcomings of the implementation of gender equality policies during the last decades are briefly pointed. Secondly, the gendered dimensions of the crisis are explored: both in the crisis genesis, in its social impacts, as well as in the policies developed to face it. A specific attention is placed in the weakening of the democratic decision-making process in key areas of the current political and economic governance. To conclude we identify the challenges, obstacles and opportunities that this
\end{abstract}


new scenario pose to mainstream gender in policy making, raising some questions regarding the need to rethink the priorities, the courses of action and the tools of equality policies.

Keywords: Economic Crisis; Equality Policies; Gender Equality; Gender mainstreaming, democratization.

\section{INTRODUCCIÓN ${ }^{1}$}

En los últimos años las políticas de género se han extendido en España y Europa: se han consolidado sus estructuras, desarrollado legislación específica, y sistematizado la elaboración de planes y programas, dotándolos de una financiación inédita. Se podría decir que nunca anteriormente se habían tenido tantas herramientas para incorporar la perspectiva de género en las políticas públicas. Aún así, los avances en incorporar el principio de igualdad de forma efectiva en el centro mismo de la actuación pública han sido, como veremos, lentos, desiguales y llenos de obstáculos.

Con la emergencia de la crisis económica y financiera global a partir de 2008 estos límites preexistentes se han situado en primer plano. La crisis ha tenido y está teniendo un gran impacto social y ha conllevado tanto notorios recortes en el gasto público como el desarrollo de reformas en profundidad en las principales políticas del Estado del Bienestar, todo ello realizado con una gran rapidez, escaso debate público y bajo la presión constante de los mercados financieros. En la Unión Europea estamos presenciando -aunque con importantes diferencias entre paísesuna transformación de carácter estructural hacia un modelo más neoliberal que evidencia, además, la existencia de una profunda crisis democrática en la que la política va perdiendo peso frente a los imperativos financieros. A su vez estos cambios en el contexto español han venido de la mano del auge de valores e ideologías conservadoras que no sólo cuestionan el rol social del Estado sino también otros derechos individuales ya reconocidos como el del aborto o el de manifestación. Ante esta situación en el Estado español se ha truncado la tendencia de los últimos años a un mayor desarrollo de las políticas de igualdad de género y a una creciente centralidad de la igualdad en la agenda política.

En este artículo nos preguntamos cómo esta crisis ha incidido en la estrategia del Gender Mainstreaming ${ }^{2}$. ¿Tiene sentido impulsar la transversalidad

\footnotetext{
${ }^{1}$ Agradecemos a Oriol Costa y Marta Otero sus valiosas aportaciones a la elaboración de este documento.

${ }^{2}$ En el artículo utilizamos indistintamente Gender Mainstreaming, sus traducciones mainstreaming de género y transversalidad de género. Sin embargo, es importante señalar que el concepto de transversalidad no acaba de trasladar adecuadamente el contenido más
} 
de género en una administración intervenida? ¿Es posible incorporar la perspectiva de género en las políticas de austeridad? ¿Qué podemos aprender de la práctica desaparición del debate acerca de la igualdad de mujeres y hombres en las respuestas a la crisis? ¿Cuál debe ser el papel del feminismo de Estado ante todo ello; cuál el de los movimientos sociales por la igualdad?

Pretendemos contribuir a estos debates sistematizando las principales tendencias de cambio, identificando los dilemas actuales de las políticas de igualdad y planteando algunas preguntas. Hemos partido del análisis de fuentes secundarias, conectando la literatura sobre políticas de igualdad con los recientes análisis de la crisis desde una perspectiva crítica y de género. Empezaremos esbozando el desarrollo de las políticas de igualdad de género a nivel europeo y caracterizando en términos generales la dimensión de género de la presente crisis económica, así como los retos y obstáculos que la gobernanza económica actual plantea a la hora de implantar la transversalidad de género. En el siguiente apartado exploramos cómo todas estas cuestiones se han concretado en el caso español. Con todo ello, finalmente discutiremos sobre la necesidad de replantear la misión y estrategias de las políticas de igualdad en este nuevo escenario para poder ir más allá de la defensa y mantenimiento de los logros conseguidos anteriormente e incidir en los enormes retos que tenemos por delante. Estos retos son, a nuestro entender, por un lado corregir la ceguera de género de las políticas socio-económicas y las reformas estructurales en marcha, y por el otro revertir el debilitamiento de los mecanismos democráticos de toma de decisiones. Sostendremos que en buena medida nos encontramos en un momento de aceleración de los cambios a muchos niveles en el que se están definiendo unas nuevas reglas del juego. Nos encontramos, pues, en un punto de inflexión y en un escenario potencialmente abierto en el cual es necesario repensar la estrategia del mainstreaming de género; para lo que se plantean en este artículo algunas reflexiones basadas en la experiencia del Estado español.

\section{POLÍTICAS DE IGUALDAD, CRISIS Y GÉNERO}

\subsection{Transversalidad de género: avances y límites a 20 años de Beijing}

El proceso de institucionalización de las políticas de igualdad de género tiene ya un notable recorrido. Inicialmente se desarrollaron iniciativas dirigidas a garantizar la igualdad de trato, en los años 80 del siglo XX políticas específicas dirigidas a promover la igualdad de oportunidades y compensar las desigualdades

sustantivo de la estrategia; esto es, la idea de que no se trata solamente de promover la cooperación horizontal sino de replantear las prioridades y objetivos integrando la perspectiva de género en la agenda política general. 
existentes, y a mitad de los noventa ${ }^{3}$ se lanzó la estrategia de la transversalidad de género para dar un nuevo paso adelante y superar las limitaciones observadas hasta el momento. Se planteó la necesidad de incidir en los mecanismos de (re)producción de la desigualdad, partiendo del reconocimiento de que las políticas públicas tienen por lo general un carácter androcéntrico, por lo que se debe revisar en profundidad su proceso de elaboración, implementación y evaluación en aras de incorporar de forma sistemática la perspectiva de género como requisito y evitar sesgos. En lo concreto, esto implica promover una nueva forma de hacer para incorporar a nuevos actores y replantear las agendas políticas con unas prioridades más inclusivas (Lombardo: 2005, Council of Europe: 1998). Esto incluye formar y sensibilizar al personal político y técnico de las administraciones, generar herramientas analíticas e informativas, revisar los objetivos de las políticas, incorporar a expertos/as, movimiento feminista y sociedad civil, transformar las culturas organizativas, expresar el liderazgo y el compromiso público con la igualdad, y crear espacios de cooperación transversal que permitan superar un acercamiento sectorial al género, entre otras cuestiones. Se trata, por lo tanto, de una estrategia de transformación en profundidad de los valores y rutinas de las administraciones públicas que de una forma más o menos explícita cuestiona y reta los valores y prioridades hegemónicas.

Este principio del Gender Mainstreaming (GM en adelante) se ha ido incorporando a los marcos normativos fundamentales a nivel nacional e internacional, entre ellos el Tratado de Ámsterdam de 1997 en la UE ${ }^{4}$. Se ha ido traduciendo también a herramientas concretas que facilitan insertar el enfoque de género en la dinámica cotidiana de las diversas políticas sectoriales, en programas específicos de acción y en la creación de estructuras institucionales dedicadas a ello.

Sin embargo esta promesa de transformación no siempre se ha materializado de la forma esperada. Las resistencias a este proceso de cambio implícitas y explícitas, intencionales o no- han sido intensas, y a la postre el compromiso con la igualdad en general no se ha considerado prioritario ${ }^{5}$. La investigación comparada sobre el despliegue del GM en diferentes contextos

\footnotetext{
${ }^{3}$ En la Cuarta Conferencia Mundial sobre la Mujer de Naciones Unidas celebrada en Beijing en 1995, donde gracias a la intensa movilización global de los movimientos feministas y de mujeres se estableció un punto de inflexión en la agenda mundial de la igualdad de género. ${ }^{4}$ En el artículo 3 (2) se establece que "En todas las actividades contempladas en el presente artículo, la Comunidad se fijará el objetivo de eliminar las desigualdades entre el hombre y la mujer y promover su igualdad".

${ }^{5}$ Para una revisión más en profundidad de la literatura sobre esta cuestión, ver Alfama y Cruells (2013), Kantola y Nousiainen (2009), Woodward (2008), o Benschop y Verloo (2006), entre otras.
} 
muestra que en la mayor parte de los países europeos los avances en su implementación no pueden considerarse sino parciales (Braithwaite: 2005); y que incluso en los países donde más se ha avanzado (Suecia y Holanda, según el análisis comparado de Megaert et al.: 2014), no podría considerarse que se haya institucionalizado totalmente ${ }^{6}$. Se ha tendido más a integrar actuaciones concretas a las políticas existentes que a transformar las prioridades y objetivos de la corriente principal de la política (Daly: 2005). También se constata que el avance en la inclusión de la igualdad de género ha sido desigual en función del ámbito sectorial: las políticas más vinculadas al área social han tendido a ser más abiertas a aplicar una estrategia de mainstreaming de género o a implantar medidas concretas de promoción de la igualdad, mientras por el contrario el núcleo duro de la política ha sido mucho más impermeable a estas iniciativas (Lombardo: 2005 y 2013).

Esto es especialmente significativo en relación a las políticas económicas, que justamente son un campo caracterizado por su ceguera al género ${ }^{7}$. Así, la falta de perspectiva de género de las políticas fiscales, macroeconómicas y de regulación del mercado laboral han provocado que los esfuerzos institucionales para favorecer la incorporación de las mujeres al empleo hayan convivido con políticas estructurales que desincentivaban esa misma participación, en una estrategia que Lina Gálvez denomina "esquizofrénica" (2013: 97) ${ }^{8}$. También las políticas de bienestar han carecido de dicha perspectiva de género. Así, Sainsbury (1996), Orloff et al. (1999), Daly y Lewis (2000), o Hobson et al.(2006) entre muchas otras autoras, han sostenido que tanto las políticas sociales como la investigación académica al respecto contienen un fuerte sesgo, demostrando que los diversos regímenes de bienestar presuponen -en mayor o menor medida- una organización social del cuidado de carácter familiarista que se basa en la explotación del trabajo de cuidados no remunerado de las mujeres.

Detrás de las dificultades existentes para implantar el GM en las políticas económicas y de bienestar encontramos de hecho una limitación epistemológica más fundamental: el reduccionismo de las perspectivas hegemónicas en el campo de

\footnotetext{
${ }^{6}$ Entendiendo la institucionalización como la incorporación de la perspectiva de la igualdad en todas las fases de todas las políticas sectoriales y en todos los niveles.

${ }^{7}$ Diferentes autoras han mostrado como las políticas macroeconómicas, laborales y comerciales de la UE han obviado la perspectiva de género, tanto en la Estrategia Europea del Empleo como en la planificación macroeconómica. Ver Lombardo (2013) y Allwood et al (2013), entre otras.

${ }^{8}$ En España De Villota (2006 y 2011) y Pazos (2013) han analizado esta cuestión en profundidad en relación a los incentivos fiscales a la inactividad.
} 
la macroeconomía ${ }^{9}$. Centradas en la esfera mercantil, conciben de forma sesgada la noción de trabajo, identificándolo con el trabajo remunerado e ignorando no sólo el trabajo de cuidados -realizado en su mayor parte por mujeres en los hogares tanto de forma no remunerada como mediante empleo informal-, sino también la dependencia del primero respecto al segundo, así como el rol clave de dicha dependencia para el sistema de producción capitalista (Carrasco, Borderías y Torns: 2011). En síntesis, los análisis macroeconómicos predominantes obvian las complejas interrelaciones existentes entre la economía formal, la informal y la doméstica, así como el rol económico que en estos ámbitos juegan mujeres y hombres (Gálvez y Rodríguez: 2012, Pérez Orozco: 2014, Benería y Floro: 2004). Esto condiciona el alcance de todas las políticas que se enmarquen en dichos análisis -ya sean expansivas o restrictivas-, y dificulta por lo tanto la inclusión de la perspectiva de género en estos campos.

Por otro lado, diferentes expertas han señalado la dificultad para implantar una estrategia de transformación de tan largo alcance como la del mainstreaming de género en un contexto eminentemente neoliberal caracterizado por el repliegue de la agenda social del Estado de bienestar y el desarrollo de nuevas formas de gobernanza de carácter multinivel y público-privado (Kantola y Nousiainen: 2009, Bacchi y Eveline: 2003, Kantola y Squires: 2012). Subrayan que aunque no siempre se haya explicitado, la estrategia del GM es una estrategia eminentemente política y democrática, no sólo porque por su propia definición implica una redefinición de la agenda de política pública de la mano del movimiento feminista, sino también porque su voluntad transformadora e inclusiva requiere necesariamente de la implicación de la ciudadanía para poder desbloquear resistencias y mover voluntades y compromisos políticos (Verloo: 2005 y Squires: 2005). Sin embargo, este carácter político y democrático con frecuencia se ha perdido en la implementación práctica de la estrategia, presentándose en mayor medida el GM desde un punto de vista técnico, como un proceso "más allá de la política", desconectado de los deseos normativos feministas y con un carácter fundamentalmente consensual (Verloo: 2005) ${ }^{10}$.

${ }^{9}$ De esta visión reduccionista no se escapan tampoco muchos de los enfoques más socialdemócratas y progresistas. En ese sentido Pérez Orozco (2011) recupera el concepto de estrabismo productivista de Antonella Picchio para referirse a este sesgo.

${ }^{10} \mathrm{La}$ implementación del GM en este contexto ha llevado a que en general se desarrollara una versión poco transformadora de la estrategia: se han abordado en mayor medida los temas tradicionales del feminismo que pueden tener una mayor compatibilidad con el marco neoliberal (como la representación política de las mujeres, la participación femenina en el mercado laboral o el emergente fomento de las mujeres emprendedoras, por ejemplo), mientras que las demandas de carácter más redistributivo y desfamiliarizador (extensión de los servicios públicos en el ámbito del cuidado, p.e.) han encontrado más resistencias. 
En el contexto actual de crisis económica estas limitaciones preexistentes se hacen más patentes, y la implementación del gender mainstreaming deviene más dificultosa, como veremos a continuación.

\subsection{Crisis y Género}

En los últimos tiempos diversas autoras han puesto de relieve las importantes connotaciones de género que tienen las crisis económicas (Gálvez: 2013; Federici: 2014; Walby: 2009a, o Elston; 2010, entre otras).

En primer lugar, se constata que las crisis se generan en procesos económicos que son desiguales en términos de género: las mujeres por lo general no están presentes en los puestos de decisión en el ámbito económico; no participan de forma igualitaria de la riqueza y el empleo; y no se suele tener en cuenta la economía y el trabajo de cuidados en las políticas económicas (Gálvez y Torres: 2010). Incluso se sugiere (Walby: 2009a, 5) que el intenso sesgo de género en el sistema financiero y su gobernanza (en cuanto a sus principios, objetivos, prácticas y al tipo de conocimiento implicado) ha tenido un papel fundamental en el estallido financiero.

En segundo lugar, es importante visibilizar que las crisis económicas tienen un impacto desigual en mujeres y hombres debido a su diferente posición socioeconómica de partida. Por nombrar sólo algunas de las cuestiones relevantes, vemos cómo en general hay más mujeres en situación de pobreza, y su inserción en el mundo laboral es más precaria (contratos a tiempo parcial, brecha salarial, segregación vertical y horizontal, etc.), con lo que en consecuencia tienen más dificultades para acceder a la protección social debido al vínculo de ésta con el empleo formal (Castro: 2013). Asimismo, con la disminución de los recursos disponibles se produce un incremento del volumen de trabajo -doméstico, de cuidados, servicios, en especial- que se asume de forma no remunerada o mediante la economía informal; en ambos casos recae desproporcionadamente en las mujeres (Pérez Orozco: 2014; Gálvez y Rodríguez: 2012; Benería y Floro: 2004).

En tercer lugar, las políticas de respuesta a la crisis contienen también importantes sesgos de género. Bettio et al. (2013), de hecho, sugieren que más que

Asimismo, la tendencia de la Nueva Gestión Pública al aumento de la auditoría, la eficiencia y la gestión basada en la evidencia, así como la emergencia de la gobernanza mixta públicoprivada ha conectado con la dimensión operativa del GM más que con la política, situando a parte del movimiento de mujeres en una posición de interlocución y cooperación con las instituciones, marginando a las voces más críticas y contribuyendo así a la despolitización de las políticas de género (Kantola y Squires 2012, Alfama y Cruells 2013). 
en los impactos directos de la crisis -como por ejemplo la disminución del empleo o del crédito- es en estas reacciones políticas donde se produce un mayor impacto negativo sobre las mujeres y sobre la igualdad de género. Las diversas investigaciones realizadas por el momento sobre la crisis actual subrayan que la perspectiva de género ha estado por lo general ausente en las respuestas a la crisis, independientemente del tipo de políticas a las que nos refiramos. Por ello es de gran interés examinar con mayor detalle qué ha sucedido en estos diversos tipos de iniciativas ${ }^{11}$.

a) Las reformas de la arquitectura y la regulación financiera, que se discutieron ampliamente en especial en la primera fase de la crisis (2008-2009). Walby (2009a) muestra como no se problematizó ni se abordó en ningún momento la dimensión de género ni en las causas de la crisis ni en sus propuestas de actuación ${ }^{12}$, y sostiene que las Recomendaciones de la Comisión Stigliz ${ }^{13}$ en 2009 serían un buen ejemplo de ello: pese a que sí incluían una preocupación por la igualdad y por la democratizaciónde la toma de decisiones en el ámbito financiero, se mantenía la ceguera al género.

b) Los paquetes de estímulo keynesianos, implantados especialmente en 2008 y 2009 como estrategia contracíclica. Por lo general también han tenido un notable sesgo de género al centrarse en el apoyo a sectores muy masculinizados de la construcción y la industria -en especial la automotriz- (Bettio et al.: 2013), como veremos posteriormente en el caso español. No se aprovecharon para fomentar la igualdad, lo que era sin duda una oportunidad única por el enorme volumen de recursos movilizados ${ }^{14}$.

c) En relación al colapso de las entidades financieras, la principal respuesta de los gobiernos e instituciones internacionales ha sido rescatar a estas entidades con dinero público. Estos rescates, de un volumen más que significativo, han ido por lo general de la mano del establecimiento de ciertas condicionalidades en cuanto a los cambios y actuaciones a realizar por estas entidades. Walby (2009a)

\footnotetext{
${ }^{11}$ Tipología definida a partir de Walby (2009a) y Ortiz y Cummins (2013).

${ }^{12}$ Dicha dimensión se concretaría por ejemplo en la exclusión de las mujeres -y de los sectores más afectados en general- en la toma de decisiones o la existencia de dinámicas generizadas en masculino en las prácticas financieras, como por ejemplo la propensión al riesgo o la visión cortoplacista. Ver también Young et al (ed) (2011).

${ }^{13}$ Comisión de Expertos del Presidente de la Asamblea General de las Naciones Unidas sobre las Reformas del Sistema Monetario y Financiero Internacional.

${ }^{14} \mathrm{El} \mathrm{80 \%} \mathrm{de} \mathrm{los} \mathrm{países} \mathrm{analizados} \mathrm{en} \mathrm{un} \mathrm{reciente} \mathrm{estudio} \mathrm{comparativo} \mathrm{(144} \mathrm{en} \mathrm{total)} \mathrm{aumentó}$ el gasto en la primera etapa de la crisis para implantar estímulos fiscales, con un crecimiento medio que llegó hasta el 3,9\% del PIB. (Ortiz y Cummins: 2013)
} 
destaca que excepto en raras ocasiones, no se han incluido criterios de género -o incluso sociales- en estas condiciones ${ }^{15}$.

d) Finalmente tenemos las políticas deflacionistas y de ajuste fiscal implementadas en Europa a partir de 2010 como respuesta al crecimiento del déficit público y a la crisis de la deuda soberana de la periferia europea. Estas políticas, también llamadas de austeridad, se han centrado en la reducción del gasto públicocomo vía para equilibrar las cuentas públicas y han ido acompañadas de reformas estructurales y de liberalizaciones para generar confianza en los mercados financieros. Estos ajustes han supuesto entre otras cosas reformas fiscales, laborales y de las pensiones, privatización de empresas públicas, y privatización o reducción de servicios públicos, incluyendo la disminución de su personal y los recortes en su salario (VVAA: 2014, Gálvez: 2013). En la medida que buena parte de esta reducción se da en los servicios de los que las mujeres son potenciales trabajadoras, usuarias o posibles proveedoras sustitutas, se está incidiendo negativamente en la igualdad entre mujeres y hombres, perpetuando las desigualdades existentes y creando otras nuevas (EWL 2012) ${ }^{16}$. Sin embargo, no sólo la perspectiva de género ha estado ausente en los principales mecanismos de implantación de estas medidas $^{17}$, sino que incluso en el amplio debate público que se ha generado acerca de sus repercusiones se ha prestado poca atención a la dimensión de género (Bettio et al.: 2013).

Más allá de estas consideraciones acerca de los sesgos de las diferentes respuestas políticas a la crisis, el modo como ésta se ha gestionado constituye en sí mismo un fuerte obstáculo para la implementación de la estrategia del Gender Mainstreaming. Se ha hecho patente el déficit de gobernanza económica democrática de la Unión Europea, tanto en su arquitectura institucional -en relación a la capacidad de incidencia de los países periféricos y sus representantes electos en la UE - como desde un punto de vista ciudadano -de capacidad de decisión de la ciudadanía y de rendición de cuentas de las decisiones-. La centralidad de la lógica de la deuda ha sido un mecanismo clave en este proceso ${ }^{18}$. El creciente

\footnotetext{
${ }^{15}$ Por ejemplo impulsar una composición más equilibrada de los Consejos de administración y los cargos directivos de los bancos (caso de Islandia), la inclusión de metas de igualdad de género en los objetivos corporativos o la limitación de bonos y salarios de los ejecutivos.

${ }^{16}$ Aunque esta es la tendencia general, los análisis comparados de (Bettio et al.: 2013 y Karamessini y Rubery: 2013) permiten también encontrar algunas excepciones parciales a esta tendencia en determinados países o en relación a iniciativas concretas.

${ }^{17}$ Bettio et al. (2013), por ejemplo, lo muestran en relación a los Programas Nacionales de Reforma de les estados miembros de la UE.

${ }^{18}$ Por ello desde diferentes ámbitos se ha venido hablando de deudocracia para hacer referencia a este mecanismo de gobierno basado en la deuda que ha acabado generando una
} 
endeudamiento de algunos Estados europeos por la asunción de deudas del sector privado, la brusca caída de ingresos públicos y el aumento del gasto ha incidido en gran medida en la estructura institucional de la UE por la tensión entre estados deudores con necesidad de liquidez y estados e instituciones acreedoras. En un periodo muy corto de tiempo se han ido desarrollando diferentes mecanismos y procedimientos destinados a articular cómo los estados deudores deben garantizar a los acreedores el pago de la deuda, y a generar expectativas positivas en los mercados financieros y modular la oscilación de la prima de riesgo. Estos mecanismos han sido en algunos casos generales ${ }^{19}$, y en otros ad hoc dependiendo de cada país implicado. Por lo demás, no siempre han tomado un carácter formal y explícito: Pavolini et al. (2015) sugieren, por ejemplo, que la influencia de la Unión Europea en países como España o Italia se ha concretado más a través de la condicionalidad de las ayudas económicas recibidas y de la diplomacia de "trastienda" ${ }^{20}$ que por la vía de procedimientos formales de coordinación política. Sostienen asimismo que su intromisión ha aumentado a raíz de la crisis de la deuda, en relación al nivel de detalle exigido en las reformas que se deben implantar, a la rapidez del calendario de aplicación y a su seguimiento. Este tipo de lógica puede llevar a una dinámica de "democracia sin opciones" de la que advierte Krastev $(2002)^{21}$, en la que la dependencia externa lleva a que la ciudadanía pueda cambiar los gobiernos con mucha mayor facilidad de la que puede cambiar las políticas públicas, con la consecuencia de un peligroso crecimiento de la frustración con el proceso democrático.

Por lo demás, esta nueva gobernanza económica no sólo se orienta a los estados deudores sino a todos los miembros de la Unión ${ }^{22}$. Con ello se ha

transferencia de rentas de la ciudadanía a los poderes financieros y a los acreedores de la deuda pública. El concepto se populariza a raíz del documental Debtocracy que muestra las causas y los impactos de la crisis de la deuda en Grecia, véase: www.debtocracy.org.

${ }^{19}$ Los instrumentos para garantizar la estabilidad monetaria de la zona euro mediante la realización de los rescates financieros asociados a ajustes estructurales son el Mecanismo Europeo de Estabilidad -creado en 2011, y que juega un papel similar al del FMI en relación a los créditos a países de América Latina en la década de los 80- y su precursor el Fondo Europeo de Estabilidad Financiera y los Memorandos de Entendimiento. (Quién debe a quién: 2011).

${ }^{20} \mathrm{La}$ definen como las negociaciones informales que se promueven des de las instituciones comunitarias para convencer a los policy makers locales de introducir las reformas propuestas por la UE.

${ }^{21}$ Citado en Bosco y Verney (2012).

${ }^{22}$ Entre las nuevas medidas implantadas para ello destacamos: el Pacto del Euro (comienzos de 2011), el Semestre Europeo (2011), el denominado Six-pack (en vigor desde enero de 2012); el Pacto Fiscal y el Tratado de estabilidad, coordinación y gobernanza (desde 2013 para todos los Estados miembros de la UE excepto el Reino Unido y la República Checa); y 
fortalecido la incidencia europea en las políticas económicas, fiscales, laborales y de gasto público de cada país, bajo el liderazgo de unas instituciones no elegidas directamente por la ciudadanía: el Consejo Europeo, la Comisión Europea, el Banco Central Europeo y la denominada Troika Comunitaria (formada por los dos anteriores junto con el FMI). Se ha desplazado así el papel de los parlamentos nacionales y del Parlamento Europeo, ubicándose la toma de decisiones en esferas cada vez más alejadas de las personas.

En conjunto, pues, las respuestas a la crisis han sido por lo general ciegas al género en el ámbito europeo, al mismo tiempo que se han creado unos mecanismos de gobernanza que dificultan aun más la implementación del mainstreaming de género. Todo ello a pesar del rol pionero que ha tenido la UE en el impulso de la agenda de igualdad de género durante décadas.

\section{BALANCE DE LA SITUACIÓN EN ESPAÑA}

\subsection{Avances y límites de las políticas de igualdad de género}

Desde los años 80 las políticas de igualdad de género han tenido un desarrollo muy importante en nuestro país, que se ha concretado en diversas estructuras, normativas e iniciativas: se han creado Institutos de la Mujer a nivel estatal y autonómico y numerosas consejerías locales, y se han definido e implementado diversas generaciones de planes de igualdad de género. Desde que entre 1995 y 1997 se empezaron a incorporar explícitamente ejes de actuación sobre transversalidad en los planes de igualdad,esta estrategia se ha ido desplegando progresivamente, y ya en la década del 2000se aprobaron diversas leyes específicas de igualdad ${ }^{23}$.

En definitiva, se ha consolidado la igualdad de género como política sectorial y se ha avanzado en áreas clave como la violencia de género, la desigualdad en las condiciones de trabajo o la atención a la dependencia,a través del

finalmente el Two-pack (desde mayo de 2013). En líneas generales, se orientan a consolidar la supervisión financiera de los presupuestos generales y de los Programas Nacionales de Reformas de los estados miembros, agilizando los mecanismos de intervención con el objetivo de asegurar el Pacto de Crecimiento y Estabilidad y el cumplimiento de los techos de deuda y déficit.

${ }^{23}$ La Ley Orgánica 3/2007 para la igualdad efectiva entre hombres y mujeres así como doce leyes autonómicas de igualdad y regulación específica sobre informes de impacto de género. Más allá de estas medidas, se crearon también comisiones interdepartamentales e interinstitucionales, observatorios de género en materias varias -comunicación, salud, violencia, entre otras cuestiones-,y consejos consultivos y de participación (Alonso: 2013). 
desarrollo de normativa estatal ${ }^{24}$ y autonómica, junto con un notable despliegue de iniciativas locales. No obstante, los avances han sido parciales. La incidencia en las políticas económicas ha sido muy débil o incluso nula en muchos momentos: de Villota $(2006,2011)$, por ejemplo, señala el efecto regresivo del modelo impositivo actual, y Carrasco, Borderías y Torns (2011) la necesidad de que el Estado de bienestar se redefina en función de las necesidades del cuidado. También el desarrollo de la transversalidad ha sido heterogénea en diferentes Comunidades Autónomas, oscilando entre un despliegue más puntual o de baja intensidad en algunas y uno más sustantivo en otras ${ }^{25}$.

Antes del inicio de la crisis financiera en el 2008, pues, el desarrollo de las políticas de igualdad de género en nuestro país era muy notable, aunque el éxito efectivo de la estrategia del GM no puede sino calificarse como lento, limitado, parcial y desigual en función de ámbitos sectoriales, territorios y niveles administrativos; como por otra parte se ha apuntado ya que ha sucedido también en la mayor parte de los estados de la UE. Todo este desarrollo, como veremos a continuación, se ha visto afectado fuertemente en primer lugar por la implementación de las medidas dirigidas a hacer frente a la crisis; en segundo lugar, por la fuerte reducción del apoyo a las políticas de igualdad a sus estructuras, y en tercer lugar, por el impulso de medidas antifeministas.

\subsection{Crisis y género}

Las primeras respuestas del gobierno español a los efectos de la crisis se articularon en el marco del Plan E o Plan Español para el Estímulo de la Economía y el Empleo, que incluía medidas de estímulo fiscal (hasta un 2,32\% del PIB), de mejora del acceso al crédito, de estabilidad del sistema financiero y de reforma estructural (Uxó et al 2009). Uno de sus programas estrella fue un programa de inversión local ${ }^{26}$ de gran magnitud (13.000 millones) dirigido a compensar la caída del empleo,reactivar la construcción y apoyar las inversiones de los ayuntamientos.

\footnotetext{
${ }^{24}$ Nos referimos en especial a la Ley contra la violencia de género del 2004 y la Ley de Promoción de la Autonomía Personal y Atención a las personas en situación de dependencia y a las familia de 2006, sin olvidar otras iniciativas de igualdad como la legalización del matrimonio homosexual (Ley 13/2005) o la creación del Consejo para la promoción de la igualdad de trato y la no discriminación de las personas por razones de origen racial y étnico ${ }^{25}$ Ver Alonso (2013) para un análisis detallado de los avances específicos de cada Comunidad Autónoma y a nivel estatal.

${ }^{26}$ Articulado mediante el Fondo Estatal de Inversión Localy el Fondo Estatal para el Empleo y la Sostenibilidad Local.
} 
La puesta en marcha de este programa es tremendamente significativa de la debilidad de la estrategia del GM en el Estado español, especialmente teniendo en cuenta que en el momento de elaboración existían aún el Ministerio de Igualdad y la Secretaría General de Políticas de Igualdad y se estaban dando los últimos coletazos del intenso impulso a la igualdad que caracterizó las dos legislaturas del presidente socialista Rodríguez Zapatero. Ni se elaboraron los preceptivos informes de impacto de género establecidos por la Ley de igualdad de 2007 (Alarcón el al.: 2011), ni se ha encontrado ninguna referencia a los términos "igualdad", "género", o ni siquiera "mujer" en los documentos informativos ${ }^{27}$, los informes de seguimiento y de impacto territorial de los Fondos accesibles en la web del Ministerio, o incluso en un amplio informe elaborado de análisis del impacto del $\mathrm{FEIL}^{28}$. Tampoco los entes locales implicados tuvieron en cuenta el posible impacto de género diferencial a la hora de elaborar las propuestas de inversión (Alarcón y Colino: 2011 y Alarcón et al.: 2011 $)^{29}$. Finalmente, pese a que este programa permitió hacer efectivos casi 700.000 contratos de trabajo en el periodo 2009-2010, teniendo en cuenta su orientación hacia al sector de la construcción, sus beneficios previsiblemente se concentraron mayoritariamente en los trabajadores hombres ${ }^{30}$.

El giro hacia las políticas de austeridad se inició en el contexto español el 12 de mayo de 2010, cuando después de recibir importantes presiones internacionales el Presidente Rodríguez Zapatero compareció ante el Congreso para anunciar el mayor plan de recortes sociales en 30 años, por un importe de 15.000 millones de euros (el 1,5\% del PIB español) (Quién debe a quién 2011). A partir de ese momento se han sucedido sucesivos recortes del gasto público y varias reformas en profundidad del mercado laboral (2010 y 2012) y de las pensiones (2011 y 2013). Esta nueva orientación se institucionalizó con la reforma del artículo 135 de la Constitución Española en agosto de 2011 -sin referéndum y con un amplio rechazo de la sociedad civil-, estableciendo que todas las administraciones públicas deben adecuar su actuaciones al principio de estabilidad presupuestaria y que el

\footnotetext{
${ }^{27}$ Con la excepción de una referencia a uno de los proyectos financiados en un municipio, que incluía un centro de mujeres.

${ }^{28}$ Observatorio Económico Local (2010).

${ }^{29} \mathrm{El}$ análisis que realizan estos autores arroja sin embargo una valoración ambivalente: aunque las agentes de género locales valoraron a posteriori que 3/4 partes de los recursos financieros manejados por el Fondo fueron orientadas hacia líneas de gasto que en mayor o menor medida propician el bienestar de las mujeres (Alarcón et al: 2011), a la vez se constata que no se realizó un análisis de género, la variabilidad intermunicipal fue muy significativa, y la inversión en equipamientos no priorizó aquellos que más contribuyen a la igualdad (Alarcón y Colino: 2011).

${ }^{30}$ Según datos del Ministerio. No se ha conseguido acceder a datos desagregados por sexo de este volumen de contratación.
} 
pago de los créditos "gozará de prioridad absoluta"31; y se intensificó con la firma del Memorándum de entendimiento con la Comisión Europea en julio de 2012, donde se plasman las condiciones impuestas por la UE para el rescate del sector bancario español de la mano de una estricta supervisión de la Troika ${ }^{32}$.

Con los rescates de bancos y cajas de ahorros, que como ya hemos mencionado no incorporaron condicionalidades sociales y de género, se produjo un trasvase muy importante de deuda privada a deuda pública (Fresnillo: 2012), la cual hasta el estallido de la crisis se mantenía por debajo de la media europea. Hasta diciembre de 2012 se estima que se habían dedicado 1.427.355 millones de euros de fondos públicos a ayudas al sector bancario español, entre medidas de capitalización y de liquidez (PACD: 2013) ${ }^{33}$. Esto, junto con la caída de los ingresos fiscales y el gasto en desempleo y estímulos fiscales, ha llevado directamente a pasar del $41,1 \%$ del PIB de endeudamiento público en 2007 a un 101,9\% a finales de 2012 (Medialdea et al.: 2013). Se dispara así el porcentaje de recursos públicos destinados al pago de la deuda y sus intereses, de tal modo que se calcula que para el 2014 el total de recursos públicos destinados a este fin será el $24 \%$ del presupuesto, superando ya la partida destinada al pago de pensiones (Fresnillo: 2013).

Los diversos Programas Nacionales de Reforma elaborados por España desde 2011 en cumplimiento de las nuevas formas de gobernanza económica europea redundan en la misma línea de intensificación de los ajustes estructurales y de promoción de la liberalización ${ }^{34}$. Tampoco contienen referencia alguna a la necesidad de valorar el impacto de género de las actuaciones que se implanten para

\footnotetext{
${ }^{31}$ También se establece que "estos créditos no podrán ser objeto de enmienda o modificación, mientras se ajusten a las condiciones de la ley de emisión", limitando así la capacidad de negociar reestructuraciones de deuda en el futuro (Quién debe a quién: 2011).

${ }^{32}$ Aunque las condiciones del Memorándum se aplican al sistema financiero y bancario, también implican al gobierno: condicionan la política fiscal, laboral y presupuestaria al establecer los límites de déficit público y el compromiso de aplicar las recomendaciones surgidas del Semestre Europeo. Todo ello sin olvidar que se plantea la corresponsabilidad del Estado en el caso de que la banca no pueda devolver los créditos. Al día siguiente del anuncio de su firma el presidente conservador Mariano Rajoy anunció un ajuste por valor de 65.000 millones de euros en dos años, el mayor de la democracia y el cuarto desde su llegada a la presidencia (El País, 12 de julio 2012).

${ }^{33}$ Incluye el rescate europeo. Esta cifra se ha calculado a partir del análisis de fuentes oficiales, si bien estos autores subrayan que en ningún organismo público se ha informado de manera completa y global de las ayudas concedidas a las entidades bancarias.

${ }^{34}$ Con efectos a nivel estatal pero también autonómico, como muestran Del Pino et al. (2014)
} 
garantizar que tengan efectos equitativos, y de hecho ni siquiera se presentan los datos del diagnóstico desagregados por sexo (Lombardo: 2013) ${ }^{35}$.

En el conjunto de servicios públicos se ha producido una gran reducción presupuestaria y de personal ${ }^{36}$; lo que afecta especialmente a las mujeres por la feminización del sector público ${ }^{37}$. Esto ha incidido especialmente en el sector sanitario, donde además del aumento de la presión asistencial, se han introducido $\operatorname{copagos}^{38}$ y otras medidas de ahorroy se restringido el acceso ${ }^{39}$.

Las reformas del sistema de pensiones han limitado el acceso y la cuantía de las prestaciones contributivas: se ha aumentado la edad de jubilación, el periodo de cálculo de la base reguladora (de 15 a 25 años), y los años cotizados que dan acceso al $100 \%$ de la base reguladora ${ }^{40}$; regulado el coeficiente de parcialidad en el cálculo de distintas prestaciones -sin resolver el problema de las personas trabajadoras a tiempo parcial- ${ }^{41}$; e introducido un índice de revalorización y un factor de sostenibilidad para desvincular la cuantía del coste de la vida ${ }^{42}$. En conjunto esto supone una relevante pérdida de poder adquisitivo de los y las pensionistas (Gala: 2014) y previsiblemente tendrá un impacto más negativo en las mujeres $^{43}$, con trayectorias laborales más discontinuas y mayor parcialidad, y

\footnotetext{
${ }^{35}$ Esta misma autora subraya la poca presencia en todos las recomendaciones de una preocupación por el bienestar de las personas especialmente afectadas por la crisis económica, con excepción quizás de la cuestión de la mejora de las oportunidades de empleo para la juventud. Ver pp. 7-8, para un análisis de los programas 2011-2013.

${ }^{36}$ Por ejemplo la tasa de reposición del personal público establecida es del 10\% (Pavolini et al.: 2015).

${ }^{37}$ Constituyen el $77 \%$ del personal sanitario y $66 \%$ en la educación (INE 1er Trimestre de 2014).

${ }^{38}$ Los recortes en la salud pública -fundamentalmente en el ámbito local-, también han incidido en la prevención de la violencia de género y la prestación de servicios de comedor para garantizar la salud infantil.

${ }^{39}$ Con el RD de 24 de abril de 2012 la prestación sanitaria deja de ser totalmente universal.

${ }^{40}$ Reformas introducidas por la Ley $27 / 2011$.

${ }^{41}$ Reforma introducida por el RDL 11/2013.

${ }^{42}$ Ley 23/2013.

${ }^{43}$ También se han desarrollado -en la Ley 27/2011- algunos mecanismos compensatorios (nuevas figuras para la adquisición de derechos de pensión en base al cuidado para compensar la pérdida de derechos por la salida del mercado de trabajo de las mujeres con cargas familiares) pero éstos ni siquiera llegan a compensar la mayor penalización para las mujeres de esta reforma (Pazos: 2013).
} 
aumentará una brecha de género ya de por sí muy notable ${ }^{44}$ (Pazos: 2013, 2014, Gala: 2014).

El apoyo público a los cuidados, que había vivido un impulso considerable durante la década del 2000 (Pavolini et al.:2015), se reduce en gran medida. Se congela el despliegue de la Ley 39/2006 de Autonomía Personal y se modifican algunos aspectos relevantes ${ }^{45}$, a la vez que se reducen las plazas públicas para la escolarización de menores de 3 años $^{46}$ y las ayudas a las familias ${ }^{47}$. Además disminuye drásticamente la financiación a los servicios sociales locales y se reforman los gobiernos locales, a través de la Ley 27/2013 de racionalización y sostenibilidad de la Administración Local.El menor gasto en políticas sociales de proximidad impacta así en un sector económico fuertemente feminizado y traslada al sector doméstico y/o informal la realización de muchas de estas tareas, aumentando la carga de trabajo de las mujeres (Gálvez y Rodríguez: 2012, Pérez Orozco: 2011).

Respecto a las politicas recaudatorias se han incrementado los impuestos indirectos ${ }^{48}$, se ha aumentado el copago, las tasas para servicios básicos gratuitos (judiciales, por ejemplo) y las tarifas en servicios subvencionados como el transporte colectivo. En tanto que mecanismos que se aplican independientemente del nivel de renta, tienen un impacto regresivo; lo que se suma a un sistema impositivo sobre la renta que desincentiva la participación de las mujeres en el mercado de trabajo y que no ha sido mejorado en las sucesivas reformas fiscales (De Villota: 2011, Pazos: 2013).

Además de las citadas medidas de ahorro público, las sucesivas regulaciones del mercado laboral han flexibilizado las condiciones de trabajo, rebajado el coste del despido y debilitado la negociación colectiva ${ }^{49}$. Todo ello ha añadido más precariedad y parcialidad a las ya de por sí peores condiciones

\footnotetext{
${ }^{44}$ La pensión media de las mujeres es el $60 \%$ de la de los hombres, según Estadísticas del Ministerio de Empleo y Seguridad Social.

${ }^{45}$ Como la exclusión de las cuidadoras familiares de la cotización a la Seguridad Social y la eliminación de niveles de la valoración de la dependencia.

${ }^{46}$ Supresióndel programa Educa 3.

47"Cheque-bebé", ayudas a las madres trabajadoras y no extensión de la baja intransferible de paternidad prevista en la Ley 3/2007 de igualdad.

${ }^{48}$ Modificación de tipos del IVA de la Ley 14/2012.

${ }^{49}$ Con la Ley 3/2012, aunque también se han implantado otras modificaciones de menor alcance, con el RD 2/2012 -que da un mayor peso a las ETT- y el RD 4/2013 de medidas de apoyo al emprendedor, que introduce el nuevo contrato a tiempo parcial con vinculación formativa (los llamados minijobs) para las personas jóvenes.
} 
laborales de las mujeres y obstaculizado los esfuerzos realizados anteriormente para incorporar medidas que favorezcan la igualdad de género en los convenios.

Por lo que respecta a las políticas especificas de igualdad, han vivido en su conjunto una notable disminución presupuestaria y de recursos humanos, además de una pérdida de peso político -marcada por la desaparición del Ministerio de Igualdad ya en 2010- y de competencias -en el caso del ámbito local, ya que la Ley 27/2013 les retira la capacidad para actuar-. En el periodo 2010-2014 el presupuesto estatal ha disminuido un $56,7 \%{ }^{50}$ sin contar con la reducción también de los recursos destinados a la violencia de género. Paleo y Alonso (en este número) aportan datos al respecto y evidencian asimismo diferencias entre Comunidades Autónomas en función del color político.

Este factor ideológico debe ser analizado desde el prisma del desarrollo de una agenda política por parte del Partido Popular -en el gobierno estatal y en la mayoría de autonomías- que podemos calificar de antifeminista, que se traduce en una vuelta a un modelo de familia tradicional y a un rol de las mujeres como esposas, madres y cuidadoras. Las medidas emprendidas en esta dirección son varias. La más controvertida es sin duda el ya retirado ${ }^{51}$ Anteproyecto de Ley de la Protección del Concebido y de los Derechos de la Embarazada que restringía el derecho a la interrupción del embarazo en casi cualquier circunstancia y suponía un grave retroceso en los derechos sexuales y reproductivos incluso respecto a la ley de 1985. También se pueden mencionar, no obstante, otras propuestas como la retirada de los anticonceptivos de última generación de los medicamentos subvencionados por la Seguridad Social (Platero: 2014), el recorte en dependencia ya mencionado, el desmantelamiento de la red de atención de violencia de género (Ruiz: 2014), la restricción del acceso de mujeres solas y parejas de lesbianas a los tratamientos de reproducción asistida de la cartera común básica pública ${ }^{52}$ (Platero: 2014), el regreso a un marco discursivo de la violencia doméstica en las declaraciones de las responsables de igualdad, o la aprobación de nuevas leyes de igualdad centradas en las mujeres embarazadas a nivel autonómico (Paleo y Alonso: en este número).

En definitiva, la crisis económica y el paso a gobiernos conservadores que se ha producido en los últimos años (a nivel estatal y en muchas autonomías) han afectando sustancialmente no sólo a los derechos sociales sino también a la igualdad entre mujeres y hombres y en particular a las políticas de género,visibilizando la fragilidad de los avances conseguidos. Desde la perspectiva temporal de los últimos 30 años, Bustelo (2014) resalta sin embargo que por el

\footnotetext{
${ }^{50}$ Información extraída de los PGE.

${ }^{51}$ En septiembre de 2014.

${ }^{52}$ El País, 18 de julio de 2013.
} 
momento en lo esencial el feminismo de Estado ha conseguido sobrevivir -con menor peso institucional y recursos, eso sí-, y que persiste un importante legado en cuanto a discursos y herramientas de política desarrolladas en años anteriores fundamentalmente normativas y estructuras de implementación-, En cualquier caso, el debate sobre si estamos en un momento de paralización temporal de las políticas de igualdad o bien de desmantelamiento y/o de un cambio más profundo en el compromiso con la igualdad y con la función social del Estado está abierto ${ }^{53}$. Será necesario continuar investigando en los próximos años para poder dar respuesta a esta cuestión.

\section{3. ¿Y AHORA QUÉ? LA TRANSVERSALIDAD DE GÉNERO EN LA ENCRUCIJADA}

En las páginas precedentes se ha recurrido a la literatura feminista sobre las políticas de igualdad y sobre la crisis económica para identificar algunos de los principales retos y límites con los que se encuentra la estrategia del Gender Mainstreaming en el contexto actual. Recapitulando, en anteriores apartados se ha argumentado que la transformación efectiva de la agenda política de respuesta a la crisis para introducir prioridades más inclusivas y más justas se encuentra con tres limitaciones de tipo general.

En primer lugar, el propio sesgo de las miradas predominantes en la economía, que al centrarse en el ámbito financiero y productivo dejan fuera del foco la economía de cuidados y doméstica y por lo tanto no son capaces de ver y plantear intervenciones en estas dos esferas fundamentales en las que se está reaccionando a los efectos de la crisis (Pérez Orozco: 2014). En segundo lugar, y conectado con lo anterior, la impermeabilidad de las políticas socio-económicas a la perspectiva de género en general y a la estrategia del Gender Mainstreaming en particular. Si ya en el periodo previo la incidencia era complicada, con el estallido de la crisis económica se ha hecho patente la consideración de las políticas de género como accesorias, de las que prescindir cuando aparece la necesidad de tomar "grandes decisiones"en el terreno económico, pretendidamente neutro ${ }^{54}$. Asimismo, la pérdida paralela de centralidad en los últimos años de las consideraciones de

\footnotetext{
${ }^{53}$ Ver, por ejemplo, muchos de los artículos recogidos en este monográfico, o desde una mirada comparada, Karamessini y Rubery (2013).

${ }^{54} \mathrm{De}$ hecho, el recorte o devaluación de la cuestión de la igualdad de género ha sido con frecuencia justamente un mecanismo para indicar y comunicar la "seriedad" con la que se abordan las cuestiones económicas. Véase como ejemplo el caso de la remodelación del Gobierno en octubre de 2010 en la que se suprimió el Ministerio de Igualdad convirtiéndose en Secretaría de Estado- con el argumento del ahorro presupuestario aunque sus efectos en este sentido eran muy limitados,
} 
justicia social -y por extensión de las políticas sociales en sentido amplio- en la agenda política no hace sino reforzar esta invisibilización de la igualdad de género. El tercer límite lo encontramos en el debilitamiento de los mecanismos democráticos de toma de decisiones en ámbitos claves, en detrimento de un peso creciente de los intereses económicos y de espacios decisorios con poca o nula rendición de cuentas a la ciudadanía. Aunque éste no es un fenómeno nuevo, sin duda en el momento actual se ha acelerado y ha adquirido dimensiones inéditas en Europa mediante la dinámica de la deuda; que en España además ha ido acompañado de la implantación de medidas crecientemente represivas para hacer frente al malestar social generado por la crisis y por las políticas de austeridad.

Estas limitaciones, no obstante, coexisten con algunas fortalezas significativas desde las cuales consideramos que se pueden (re)construir las estrategias políticas por la igualdad a corto y medio plazo.

Por un lado, la trayectoria de las políticas de igualdad en los últimos años es un factor muy relevante que debe ser tenido en cuenta. Sin duda, las numerosas estructuras de igualdad creadas en todos los niveles de gobierno, los múltiples programas implementados, el gran volumen de personal técnico y político de las administraciones sensibilizado y formado en igualdad, o los nuevos mecanismos y herramientas creadas, por citar sólo algunos elementos, nos sitúan en un escenario nuevo. Pese a la falta de recursos actual, la evaporación de la igualdad de género en la agenda institucional e incluso en los casos donde se están poniendo en marcha unas agendas políticas conservadoras y/o antifeministas, no cabe pensar que todo este despliegue haya sido en vano. Incluso es posible afirmar que nunca anteriormente se habían tenido tantas herramientas para incorporar la perspectiva de género en las políticas públicas ni tanta conciencia del sesgo de género existente. En particular, los y las agentes de igualdad -u otros/as actores/as especializados/asde las administraciones públicas son un recurso muy valioso, al mantener cotidianamente una preocupación por la igualdad en su trabajo diario, tenga éste o no una vinculación explícita con las políticas de género. También a nivel social se ha avanzado en gran medida en la valoración de la igualdad de género como un componente fundamental del modelo democrático y social, como evidencia la evolución de los datos acerca de las percepciones y actitudes sociales en relación a estas cuestiones, e incluso la mayor -aunque aún no sistemática- incorporación de la perspectiva de género en algunas de las movilizaciones sociales recientes ${ }^{55}$.

Por otro lado, el momento actual no sólo plantea obstáculos sino también oportunidades que es importante señalar. En el contexto español en particular, el

\footnotetext{
${ }^{55}$ Ver Cruells y Ruiz (2014) para un análisis del 15M.
} 
deterioro de la situación económica ha ido de la mano de una profunda crisis institucional y política, que ha generado un extendido reclamo democratizador. Crece la problematización de la cultura política y organizativa predominante en las instituciones públicas y la demanda de abrir los procesos de decisión y hacerlos más transparentes, cercanos y permeables a la ciudadanía para poder discutir y replantear las prioridades en el marco de una agenda social amplia. Esto sin duda conecta con la apertura, democratización y politización que plantea la estrategia del Gender Mainstreaming. A su vez, el movimiento feminista parece haberse revitalizado y rejuvenecido a raíz de las últimas iniciativas conservadoras impulsadas por el gobierno de Mariano Rajoy (Bustelo, 2014).

Desde nuestro punto de vista, pues, el momento actual plantea retos importantes que exigen de una revisión de la misión, estrategia e instrumentos de las políticas de igualdad, del propio feminismo de Estado y en particular de la estrategia del mainstreaming de género. En este contexto pueden ser identificados tres niveles principales de actuación de los organismos de igualdad.

En el primero se pondría el foco en la contención y en la defensa y mantenimiento de lo ya existente: las estructuras, presupuestos y servicios de promoción de la igualdad así como los procesos de incorporación de la perspectiva de género en las políticas sectoriales (mediante por ejemplo, la continuidad en la formación del personal público, la elaboración de informes de impacto, etcétera); lo que permite consolidar y dar continuidad en la medida de lo posible a los avances previos. Así, se continúa aplicando el GM pero de una forma que podríamos denominar periférica: en programas o iniciativas puntuales, y con frecuencia en los espacios o temas que presentan menos resistencias. Obviamente, los recortes en las estructuras de igualdad o su pérdida de peso en los organigramas pueden afectar en gran medida a la capacidad de estos actores de ir más allá de este nivel de actuación. Sin negar la gran importancia de intentar mantener los logros conseguidos, sostenemos que los cambios han sido tan profundos y el impacto de la crisis es tan notable que este tipo de intervención del feminismo institucional pierde sentido si no se enmarca en una reflexión sobre cuál es y debe ser a día de hoy la agenda política de la igualdad y la consiguiente escala de prioridades. En este sentido, sería importante valorar empíricamente hasta qué punto es en este nivel, como pareciera que sucede mayoritariamente en el caso de España ${ }^{56}$, donde se concentra buena parte de la actividad de los organismos de igualdad en la actualidad

\footnotetext{
${ }^{56}$ Aquí habría que tener en cuenta sin embargo la heterogeneidad territorial característica del desarrollo de las políticas de género en España, que lleva a que las dinámicas de las Comunidades Autónomas en cuanto al mantenimiento o retirada del apoyo público a la igualdad hayan sido diferentes (Paleo y Alonso: en este número).
} 
y cuáles serían en todo caso los factores que permitirían desarrollar estrategias más ambiciosas.

Un segundo nivel, más sustantivo, se enfocaría en incidir enla negociación de las políticas clave. En el momento actual esto implica centrarse en introducir la perspectiva de género en las medidas de austeridad que se están llevando a cabo así como en las profundas reformas estructurales en marcha (de los servicios públicos vinculados a derechos básicos, de las pensiones, del régimen local, laborales, etcétera); todas ellas reformas de gran calado por las que no sólo se reduce el gasto sino también se modifican sustancialmente las bases del Estado de bienestar español, los marcos de relaciones socioeconómicas y el propio rol de los poderes públicos. En este sentido, para poder realmente incidir en el núcleo principal de las políticas que más están impactando en la ciudadanía probablemente habría también que replantear las prioridades políticas y la visión estratégica de los organismos de igualdad y su relación con los gobiernos de los que forman parte. A lo largo del artículo se ha hecho referencia a las diversas investigaciones que muestran cómo el análisis y la sensibilidad de género ha estado mayoritariamente ausente en las respuestas a la crisis tanto a nivel europeo como español, pese a la rápida extensión previa del GM. A la luz de los resultados, pues, el peso político y la capacidad de los organismos de igualdad españoles para incidir en estas medidas clave ha sido limitada. Además, el debilitamiento generalizado -si bien no homogéneo en todo el territorio- del feminismo institucional supone una dificultad central. En este marco, ¿cómo pueden estos actores acceder a los espacios de decisión relevantes, en un contexto marcado por las prisas y por la desaparición del discurso de la igualdad? ¿Quieren y pueden los organismos de igualdad jugar este papel de vehicular en los espacios de decisión económico-política las demandas sociales de las mujeres y de los movimientos feministas? ¿Qué sería necesario para ello? ¿Qué podemos aprender de los éxitos y fracasos de las experiencias de otros contextos y países?

En este punto nos preguntamos también qué alcance puede tener este tipo de incidencia en las políticas anticrisis y las reformas estructurales en un contexto donde la agenda política principal -y sus marcos interpretativos- tienen ya un profundo sesgo androcéntrico y antisocial. Aun suponiendo que se pudiera efectivamente aplicar la perspectiva de género a las políticas de austeridad ¿sería esto suficiente? ¿no son en la práctica dos nociones sustancialmente antagónicas?

Por lo demás, esta mesa de negociación y decisión a la que nos referíamos se desplaza cada vez más al margen de los canales políticos democráticos. Y aquí es donde situaríamos el tercer nivel de intervención política, que atañe a los dos aspectos (sustantivo y democrático) señalados a lo largo de este artículo: por un lado se trata de abordar el debate acerca de cómo modificar la agenda política, redefiniendo las prioridades sociales y la conceptualización de lo económico, y por 
el otro de incidir en la recuperación, fortalecimiento y apertura de los espacios democráticos de toma de decisiones. Recordemos que la estrategia del Gender Mainstreaming contiene estas dos dimensiones y, de hecho, buena parte de la reflexión académica acerca de su implementación gira alrededor de su capacidad de transformación efectiva -de cumplir su potencial revolucionario, en palabras de Verloo (2005)-. No obstante, modificar la agenda necesariamente deviene más complejo cuando cada vez más las decisiones se toman fuera de los espacios democráticos públicos, que son donde se ha desplegado el mainstreaming de género. En esta dirección, parece ineludible que si se quiere provocar un cambio de agenda una prioridad fundamental debería ser recuperar los espacios democráticos de decisión en los que se rinde cuentas a la ciudadanía y se eligen colectivamente las prioridades públicas.

Esto dependerá sin duda de la correlación de fuerzas existente y de que haya suficientes energías sociales sosteniendo este proyecto de radicalización democrática. De este modo, la oportunidad que ofrece la actual expansión en España de organizaciones y movimientos de la sociedad civil -e incluso nuevos partidos políticos ${ }^{57}$ - en demanda de una mayor democratización y de un cambio de agenda podría ser aprovechada también desde el feminismo institucional para fortalecer los vínculos con el conjunto de agentes sociales, revisando así de forma amplia la idea del "triángulo de terciopelo" ${ }^{58}$. Evidentemente, ni estas alianzas están exentas de conflicto ni pueden ser lideradas desde las instituciones pero es innegable que el apoyo a las iniciativas de empoderamiento ciudadano, politización y apertura de espacios críticos debe ser uno de los elementos clave de la agenda de la igualdad.

Para finalizar, consideramos que es importante tener en cuenta que nos encontramos en un momento potencialmente abierto. Tal y como lo señala Walby (2009b) las crisis son momentos de redistribución del valor, potenciales puntos de inflexión hacia tiposdiferentes de sociedad ${ }^{59}$; y en particular son momentos clave en los que las normas de género se ven reforzadas, modificadas o transformadas (Elston: 2010, Gálvez y Rodríguez: 2012). En el caso actual, hoy la agenda política parece estar siendo cuestionada, y han emergido nuevos actores y nuevas prácticas, pero sin duda aún está por ver hasta qué punto la salida de la crisis va a resultar en un cierre oligárquico que profundice las desigualdades y la falta de democracia o

\footnotetext{
${ }^{57}$ Sería el caso de Podemos o de las diversas candidaturas municipalistas surgidas a raíz de la convocatoria electoral de elecciones locales de mayo de 2015.

${ }^{58}$ Woodward (2004).

${ }^{59} \mathrm{Si}$ bien, como señalaba Klein (2007) estos momentos de inflexión han sido utilizados como oportunidades de reestructuración y cambio en el sentido neoliberal, también pueden ser oportunidades de cambio en otra dirección.
} 
bien en un cambio de reglas del juego que ponga el foco en la justicia social. En la medida en que estamos viviendo uno de estos puntos de inflexión, es necesario pensar puede o debe ser el rol del mainstreaming de género, de las feministas institucionales que lo gestionan y de los movimientos sociales que promueven estos objetivos transformadores.

La revisión de la literatura muestra que queda mucho por investigar de forma comparada acerca de los impactos de género de la crisis, de su gestión por parte de las políticas públicas, y de la fortaleza de los cambios sociales conseguidos gracias a casi 20 años de transversalidad. En particular, sería necesario analizar empíricamente hasta qué punto el feminismo institucional en diferentes contextos ha optado por el primer, el segundo y/o el tercer nivel de actuación en este momento de encrucijada; y en cada uno de ellos valorar qué estrategias, instrumentos y alianzas se han adoptado, y cuáles han resultado más o menos útiles para la consecución de una mayor igualdad entre hombres y mujeres y de un mayor peso efectivo de las voces feministas en los procesos democráticos de toma de decisión. En cualquier caso, este tipo de indagaciones serán muy útiles para reflexionar y afinar en las estrategias de actuación de los próximos años.

\section{BIBLIOGRAFÍA}

ALARCÓN, GLORIA, COLINO, JOSÉ (2011): La perspectiva de género en los gastos en infraestructuras públicas: los equipamientos educativos y deportivos en el FEIL-2009. Presupuesto y Gasto público, ${ }^{\circ}$ 64, 155-78.

ALARCÓN, GLORIA, ARIAS, CAROLINA, Y COLINO, JOSÉ (2011): Infraestructuras y género, análisis del FEIL-2009: población municipal y CCAA. Investigaciones Feministas, vol 2, 151-174.

ALFAMA, EVA y CRUELLS, MARTA (2013): How can evaluation contribute to the Gender Mainstreaming strategy? Ponencia presentada en el 3rd European Conference on Politics and Gender (ECPG). Barcelona, España.

ALLWOOD, GILL, GUERRINA, ROBERTA AND HEATHER MACRAE (ed) (2013): Unintended consequences of EU policies - Reintegrating gender in European studies. Women's Studies International Forum, Volume 3962 (JulyAugust 2013).

ALONSO, ALBA (2013): El mainstreaming de género en España. Cronología, instrumentos e impacto en las políticas finales. Tesis doctoral, Universidad de Santiago de Compostela.

BACCHI, CAROL Y EVELINE, JOAN (2003): Mainstreaming and neoliberalism: a contested relationship. Policy and Society, 2003; 22 (2), 98-118.

BENSCHOP, YVONNE AND VERLOO, MIEKE (2006) 'Sisyphus' Sisters: Can Gender Mainstreaming Escape the Genderedness of Organizations?, ournal of Gender Studies, 15:1,19-33. 
BRAITHWAITE, MARY (2005): Gender-sensitive and women friendly public policies: a comparative analysis of their progress and impact. Informe final del Proyecto EQUAPOL (2002-2005). Luxembourg: Office for Official Publications of the European Communities.

BENERÍA, LOURDES Y FLORO, MARÍA S. (2004): Deconstructing Poverty, Labor Market Informalization, Income Volatility and Economic Insecurity in Bolivia and Ecuador. Ginebra: UNRISD Background Paper.

BETTIO, FRANCESCA, CORSI, MARCELLA, D'IPPOLITI, CARLO; LYBERAKI, ANTIGONE,SAMEK, MANUELA y VERASHCHAGINA, ALINA (2012): The impact of the economic crisis on the situation of women and men and on gender equality policies. Synthesis Report. European Commission, Directorate General for Justice. Luxembourg: Publications Office of the European Union.

BOSCO, ANNA Y VERNEY, SUSANNAH (2012): Electoral Epidemic: The Political Cost of Economic Crisis in Southern Europe, 2010-11. South European Society and Politics, Volume 17, Issue 2, pp 129-154

BUSTELO, MARÍA (2014): Three Decades of State Feminism and Gender Equality Policies in Multi-governed Spain. Sex Roles, June 2014. doi:10.1007/s11199014-0381-9

CARRASCO, CRISTINA, BORDERÍAS, CRISTINA, Y TORNS, TERESA (EDS.) (2011): El trabajo de cuidados: Historia, teoría y políticas. Madrid: La Catarata.

COUNCIL OF EUROPE (1998): Conceptual Framework, Methodology and Presentation of Good Practices: Final Report of Activities of the Group of Specialists on Mainstreaming. EG-S-MS. Strasbourg: Council of Europe.

CRUELLS, MARTA AND RUIZ, SONIA (2014) "Political intersectionality within the Indignados social movement" Research on Social movements, conflicts and change, 37.

DEL PINO, ELOÍSA, RAMOS, JUAN A., y BLANCO, FRANCISCO A. (2014): Crisis fiscal, austeridad y políticas sociales: iniciativas de ajuste en siete Comunidades Autónomas. En Congreso CABISE'14 Revaluando el Capitalismo de Bienestar en el Sur de Europa: Presente y futuro del Estado de Bienestar en España. Universidad de Oviedo, 5 y 6 de junio de 2014, Oviedo, España.

DE VILLOTA, PALOMA (2006): Impacto de la política fiscal en la igualdad de género, estructura impositiva, gastos e ingresos. En De la Fuente, M. y Ortiz, L. (coords) Els pressupostos de gènere, repte per als governslocals, 49-84. Barcelona: Institut de Ciències Polítiques i Socials.

DE VILLOTA, PALOMA (2011): El impacto de la crisis económica en los tributos y en la sostenibilidad del nuevo modelo social en España: Aproximación desde una perspectiva de género. Investigaciones feministas (2), 71-94.

DALY, MARY (2005): Gender Mainstreaming in Theory and Practice. Social Politics, 12(3), 433-450.

DALY, MARY y LEWIS, JANE (2000): The concept of social care and the analysis of contemporary welfare states". The British journal of sociology, 51(2), 281-298. 
EUROPEAN WOMEN'S LOBBY (2012): The price of austerity. The impact on women's rights and gender equality in Europe. Brussels: European Women's Lobby.

FEDERICI, SILVIA (2014): Revolución en punto cero: trabajo doméstico, reproducción y luchas feministas. Madrid: Traficantes de Sueños.

FRESNILLO, IOLANDA (2012): Auditoría ciudadana. Una herramienta para revertir la "deudocracia". Viento sur, Número 123 /Junio 2012.

FRESNILLO, IOLANDA (2013): La deudocracia como amenaza global. Barcelona: Observatori del Deute en la Globalització.

GALA, CAROLINA (2014): Últimas reformas en materia de pensiones". Ponencia presentada en la jornada El Gènere de la Troika. Observatori IQ, 21 de marzo de 2014, Barcelona.

GÁLVEZ, LINA (2013): Una lectura feminista del austericidio. Revista de Economía Crítica, $\mathrm{n}^{\mathrm{o}} 15$ (primer semestre 2013), 80-110.

GÁLVEZ, LINA Y TORRES, JUAN (2010): Desiguales: mujeres y hombres en la crisis financiera. Barcelona: Icaria.

GÁLVEZ, LINA Y RODRIGUEZ, PAULA (2012): La desigualdad de género en las crisis económicas. Investigaciones Feministas, 2, 113-132.

HOBSON, BARBARA, LEWIS, JANE Y SIIM, BIRTE (2002): Contested concepts in gender and social politics. Edward Elgar Publishing.

KANTOLA, JOHANNA AND NOUSIAINEN, KEVÄT (2009): Institutionalising Intersectionality in Europe: Legal and Political Analyses, International Feminst Journal of Politics, 11 (4). 459-477

KANTOLA, JOHANNA AND SQUIRES, JUDITH (2102): From State Feminism to Market Feminism?. International Political Science Review, 33 (4) $382-400$.

KARAMESSINI, MARIA AND RUBERY, JILL (ed) (2013): Women and austerity: the economic crisis and the future for gender equality. London: Routledge.

KLEIN, NAOMI (2007): La doctrina del shock: el auge del capitalismo del desastre. Barcelona: Paidós Iberica.

LEÓN, MARGARITA Y LOMBARDO, EMANUELA (2014): Igualdad de género y políticas sociales. CIS

LOMBARDO, EMANUELA (2005): Integration or Setting the Agenda? Gender Mainstreaming in the European Constitution Making Process. Social Politics, 12(3), 412-432. http://dx.doi.org/10.1093/sp/jxi022

LOMBARDO, EMANUELA (2013): Gender mainstreaming and policy responses to the economic crisis: the 'unintended consequences' of EU and national policymaking on Spanish gender equality policies. Ponencia presentada en la Conferencia AECPA, Sevilla 18-19 septiembre de 2013.

MEDIALDEA, BIBIANA, ÁLVAREZ, IGNACIO, FRESNILLO, IOLANDA., LABORDA, JUAN, Y UGARTECHE, OSCAR (2013): Qué hacemos con la deuda Madrid: Akal.

MEGAERT, LUT, VERLOO, MIEKE AND BLEIJENBERGH, INGE (2014): Sustainability of gender mainstreaming: the effect of different levels of 
institutionalization. Ponencia presentada en Conferencia de Primavera de Atgender. Barcelona, 25-27 de junio 2014.

MINISTERIO DE EMPLEO Y SEGURIDAD SOCIAL (2012): Anuario 2012 de Estadísticas. Acceso en

http://www.empleo.gob.es/estadisticas/ANUARIO2012/welcome.htm

MINISTERIO DE POLÍTICA TERRITORIAL (2010): Informe de seguimiento del Fondo Estatal de Inversión Local (FEIL). Julio de 2010.

OBSERVATORIO ECONÓMICO LOCAL (2010): Análisis del impacto del Fondo Estatal de Inversión Local (FEIL). España y Comunidades Autónomas. Barcelona: Diputació de Barcelona.

ORLOFF, ANNE, O'CONNOR, JULIA Y SHAVER, SHEILA (1999): States, Markets, Families: Gender, Liberalism and Social Policy in Australia, Canada, Great Britain and the United States. Cambridge University Press.

ORTIZ, ISABEL Y CUMMINS, MATTHEW (2013): The Age of Austerity. A Review of Public Expenditures and Adjustment Measures in 181 Countries. Working Paper. Initiative for Policy Dialogue and The South Centre.

PALEO, NATALIA y ALONSO, ALBA (en este número): ¿Es sólo una cuestión de austeridad? Crisis económica y políticas de género en España. Investigaciones Feministas, 5.

PAVOLINI, EMMANUELE, LEÓN, MARGARITA, GUILLÉN, ANA M., y ASCOLI, UGO (2015): From austerity to permanent strain? The EU and welfare state reform in Italy and Spain. Comparative European Politics 13, 56-76 (January 2015). doi:10.1057/cep.2014.41

PAZOS, MARÍA (2014): "L'aclapadora lògica del sistema de pensions". Nous Horitzons, 206, 42-49.

PAZOS, MARIA (2013) Desiguales por ley. Madrid: La Catarata.

PÉREZ OROZCO, AMAIA (2011): Crisis multidimensional y sostenibilidad de la vida. Investigaciones Feministas, 2, 29-53.

PÉREZ OROZCO, AMAIA (2014): Subversión feminista de la economía. Aportes para un debate sobre el conflicto capital-vida. Madrid: Traficantes de Sueños.

PLATAFORMA AUDITORÍA CIUDADANA DE LA DEUDA (2013): $L a$ ilegitimidad de los Rescates a la Banca en el Estado español. Informe disponible en http://auditoriaciudadana.net/2013/10/14/presentacion-delinforme-la-ilegitimidad-de-los-rescates-a-la-banca-en-el-estado-espanol/

PLATERO, RAQUEL (LUCAS) (2014): Las políticas neoliberales contra los derechos sexuales. En Anuario de movimientos sociales 2013. Bilbao: Fundación Betiko.

QUIÉN DEBE A QUIÉN (2011): Vivir en deudocracia. Iban un portugués, un irlandés, un griego y un español... Barcelona: Icària.

RUIZ, SONIA (2014): Austerity Policy from a Feminist Perspective. The Spanish Case. Friedrich Ebert Stiftung International PolicyAnalysis.

SAINSBURY, DIANE (1996): Gender, Equality, and WelfareStates. Cambridge: Cambridge University Press. 
SQUIRES, JUDITH (2005): Is Mainstreaming transformative? Theorizing Mainstreaming in the context of Diversity and Deliberation. Social Politics, 2(3), 366-388.

UXÓ, JORGE, PAÚL, JESÚS, Y JAVIER SALINAS (2009): El programa español de estímulo fiscal frente a la crisis: justificación, características y comparación internacional. Documentos de trabajo 09/2009. Instituto Universitario de Análisis Económico y Social - Universidad de Alcalá de Henares

VERLOO, MIEKE (2005): Displacement and empowerment: Reflections on the Concept and Practice of the Council of Europe approach to Gender Mainstreaming and Gender Equality. Social Politics, Fall 2005, 344-365.

VV.AA. (2014): ¿De qué va la Unión Europea? Deuda y tratados comerciales como instrumentos del neoliberalismo y el autoritarismo. Baladre, CGT, Ecologistas en Acción, Zambra.

WALBY, SYLVIA (2009A): Gender and the financial crisis. Paper for UNESCO Project on Gender and the Financial Crisis, Lancaster University.

WALBY, SYLVIA (2009B): Contested Futures: Is the Financial Crisis a Tipping Point?. Paper for seminar: Economic Crisis and After: TheMeaning of the Present Conjuncture 29 May, London. Lancaster University, Lancaster.

WALBY, SYLVIA (2013): Finance versus democracy? Theorizing finance in society. Work, Employment \& Society, 27(3), 489-507.

WOODWARD, ALISON (2004): Building velvet triangles: Gender and informal governance, en Christiansen, T. y Piattoni, S. (eds). Informal Governance in the European Union. Cheltenham: Edward Elgar, 76-98.

YOUNG, BRIGITTE, BAKKER, ISABELLA, AND ELSON, DIANE (EDS) (2011): Questioning Financial Governance from a Feminist Perspective. London: Routledge IAFFE Advances in Feminist Economics. 